\title{
Three CRISPR-Cas immune effector complexes coexist in Pyrococcus furiosus
}

\author{
SONALI MAJUMDAR, ${ }^{1}$ PENG ZHAO, ${ }^{1}$ NEIL T. PFISTER, ${ }^{1}$ MARK COMPTON, ${ }^{2}$ SARA OLSON,${ }^{3}$ CLAIBORNE V. \\ C. GLOVER III, ${ }^{1}$ LANCE WELLS, ${ }^{1}$ BRENTON R. GRAVELEY, ${ }^{3}$ REBECCA M. TERNS, ${ }^{1}$ and MICHAEL P. TERNS ${ }^{1,4,5}$ \\ ${ }^{1}$ Department of Biochemistry and Molecular Biology, University of Georgia, Athens, Georgia 30602, USA \\ ${ }^{2}$ Department of Poultry Science, University of Georgia, Athens, Georgia 30602, USA \\ ${ }^{3}$ Department of Genetics and Genome Sciences, Institute for Systems Genomics, University of Connecticut Health Center, Farmington, Connecticut \\ 06030-6403, USA \\ ${ }^{4}$ Department of Genetics, University of Georgia, Athens, Georgia 30602, USA \\ ${ }^{5}$ Department of Microbiology, University of Georgia, Athens, Georgia 30602, USA
}

\begin{abstract}
CRISPR-Cas immune systems function to defend prokaryotes against potentially harmful mobile genetic elements including viruses and plasmids. The multiple CRISPR-Cas systems (Types I, II, and III) each target destruction of foreign nucleic acids via structurally and functionally diverse effector complexes (crRNPs). CRISPR-Cas effector complexes are comprised of CRISPR RNAs (crRNAs) that contain sequences homologous to the invading nucleic acids and Cas proteins specific to each immune system type. We have previously characterized a crRNP in Pyrococcus furiosus (Pfu) that contains Cmr (Type III-B) Cas proteins associated with one of two size classes of crRNAs and cleaves complementary target RNAs. Here, we have isolated and characterized two additional native Pfu crRNPs containing either Csa (Type I-A) or Cst (Type I-G) Cas proteins and distinct profiles of associated crRNAs. For each complex, the Cas proteins were identified by mass spectrometry and immunoblotting and the crRNAs by RNA sequencing and Northern blot analysis. The crRNAs associated with both the Csa and Cst complexes originate from all seven Pfu CRISPR loci and contain identical $5^{\prime}$ ends (8-nt repeat-derived $5^{\prime}$ tag sequences) but heterogeneous $3^{\prime}$ ends (containing variable amounts of downstream repeat sequences). These crRNA forms are distinct from Cmr-associated crRNAs, indicating different $3^{\prime}$ end processing pathways following primary cleavage of common pre-crRNAs. Like other previously characterized Type I CRISPR-Cas effector complexes, we predict that the newly identified Pfu Csa and Cst crRNPs each function to target invading DNA, adding an additional layer of protection beyond that afforded by the previously characterized RNA targeting Cmr complex.
\end{abstract}

Keywords: CRISPR; Cas; Csa; Cst; Cmr; Pyrococcus furiosus

\section{INTRODUCTION}

CRISPR-Cas systems confer prokaryotes with adaptive immunity against viruses, conjugative plasmids, and other potential genome invaders (Terns and Terns 2011; Wiedenheft et al. 2012; Reeks et al. 2013; Gasiunas et al. 2014; van der Oost et al. 2014). A host CRISPR (clustered regularly interspaced short palindromic repeats) locus contains a leader region (typically 100-500 bp) followed by multiple copies of a repeat sequence $(\sim 30-40 \mathrm{bp})$ separated by similarly sized, variable invader-derived sequences (Bolotin et al. 2005; Mojica et al. 2005; Pourcel et al. 2005; Lillestol et al. 2006; Makarova et al. 2006; Grissa et al. 2007). CRISPR RNAs (crRNAs) are produced by processing of CRISPR locus transcripts that arise from promoter elements in the leader region (Jansen et al. 2002; Tang et al. 2002). Each crRNA contains a

Corresponding authors: rterns@bmb.uga.edu,mterns@bmb.uga.edu Article published online ahead of print. Article and publication date are at http://www.rnajournal.org/cgi/doi/10.1261/rna.049130.114. guide region comprised of invader-derived sequences that allow crRNA-Cas protein effector complexes to recognize and destroy invader nucleic acids (Barrangou et al. 2007; Marraffini and Sontheimer 2008; Hale et al. 2009, 2012; Garneau et al. 2010; Jore et al. 2011; Lintner et al. 2011b; Wiedenheft et al. 2011a; Fischer et al. 2012; Gasiunas et al. 2012; Jinek et al. 2012; Westra et al. 2012a; Elmore et al. 2013; Maier et al. 2013; Mulepati and Bailey 2013; Peng et al. 2013, 2015; Sinkunas et al. 2013; Plagens et al. 2014).

CRISPR-associated (Cas) proteins provide enzymatic machinery and structural components to carry out the distinct phases of the CRISPR-Cas pathway. Moreover, modules of Cas proteins (e.g., Csa, Cst, Cse, Csm, Cmr) comprise the

(C) 2015 Majumdar et al. This article is distributed exclusively by the RNA Society for the first 12 months after the full-issue publication date (see http:// rnajournal.cshlp.org/site/misc/terms.xhtml). After 12 months, it is available under a Creative Commons License (Attribution-NonCommercial 4.0 International), as described at http://creativecommons.org/licenses/by-nc/4.0/. 
distinct CRISPR-Cas immune systems: Type I (A-G), Type II (A-C), and Type III (A-B) (Haft et al. 2005; Makarova et al. 2006, 2011; Vestergaard et al. 2014). (Note that the Cst system that is a focus of this work is variously consolidated with the Csh system into a single Type I-B immune system [Makarova et al. 2011] or defined as the distinct Type I-G immune system [Vestergaard et al. 2014].) Some individual Cas proteins from the various CRISPR-Cas systems are members of superfamilies that can have related functions in the distinct systems.

Pyrococcus furiosus (Pfu), a hyperthermophilic archaeon, has three CRISPR-Cas systems: two Type I systems, Csa (Type I-A) and Cst (Type I-G), and a relatively well-studied Type III system, Cmr (Type III-B) (Fig. 1A). While the Csa and Cst complexes have not been extensively studied, Cas5, Cas7, Cas8 (large subunit), and Cas11 (small subunit) superfamily proteins comprise other characterized Type I crRNPs (the latter sometimes fused to the large subunit as a domain) (Brouns et al. 2008; Lintner et al. 2011b; Wiedenheft et al.

A

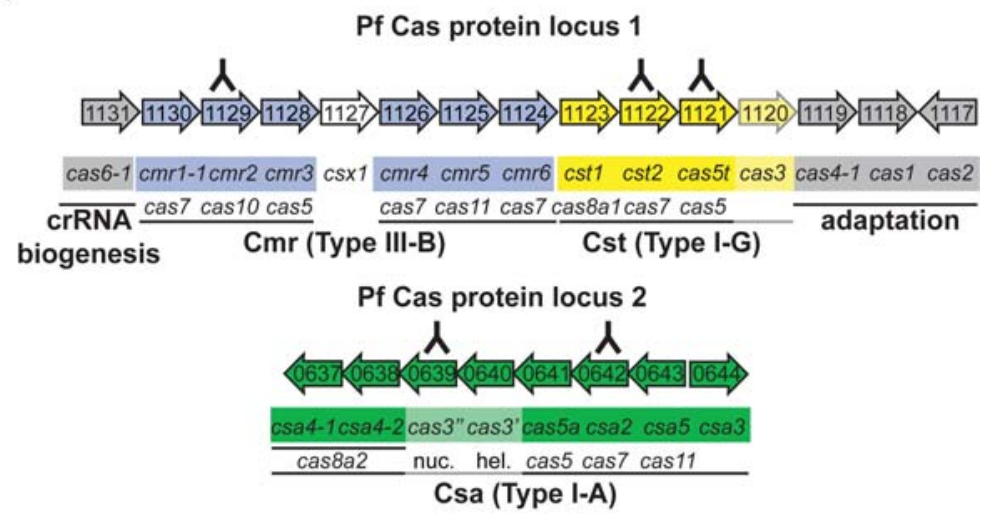

B

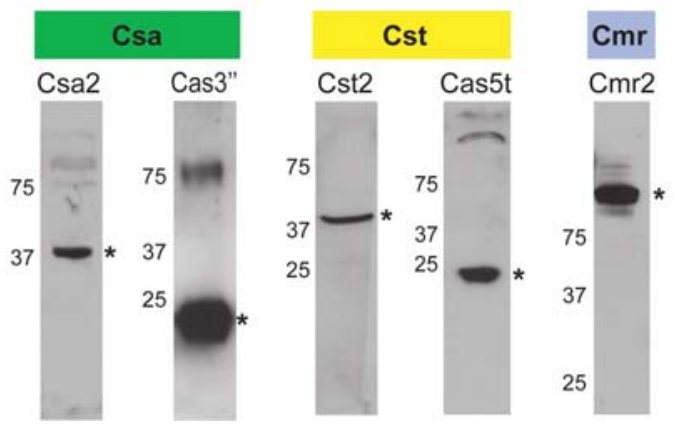

FIGURE 1. (A) Organization of cas genes in Pyrococcus furiosus. Cas genes are mainly distributed in two gene clusters in $P f u$. Genes encoding predicted adaptation Cas proteins (Cas1, Cas2, Cas4, gray), crRNA biogenesis protein (Cas6, gray) and immune-effector modules: Type I-A (Csa, green), Type I-G (Cst, yellow), Type III-B (Cmr, blue) are labeled. Genes encoding Cas3 (light yellow) and Cas3' (helicase or hel.), Cas3" (nuclease or nuc.) (light green) correspond to their subtype association. Inverted $\mathrm{Y}$ indicates polyclonal antibodies were raised against the specified Csa, Cst, and Cmr proteins. (B) Western blotting of Pfu extract with Csa, Cst, and Cmr antibodies. The indicated antibodies detect a major band (asterisk) of the expected molecular weight for each antigen. The predicted molecular weights for each protein are Csa2 $(37.4 \mathrm{kDa})$, Cas3" $(22.9 \mathrm{kDa})$, Cst2 $(39.7 \mathrm{kDa})$, Cas5t $(23.8 \mathrm{kDa})$, and Cmr2 (100.8 kDa). 2011a,b; Nam et al. 2012; Plagens et al. 2012; van Duijn et al. 2012; Brendel et al. 2014). The signature Cas3 (HD nuclease-DExH helicase) proteins of Type I systems interact with the crRNPs and cleave invader DNA (Cady and O'Toole 2011; Westra et al. 2012a; Mulepati and Bailey 2013; Sinkunas et al. 2013; Hochstrasser et al. 2014; (2014). The cas gene clusters in Pfu also encode dicted adaptation proteins (Cas1, Cas2, Cas4) and a NA biogenesis protein (Cas6) (Fig. 1A). Pfu constitutively 作 each locus having essentially the same CRISPR repeat Wang et al. 2011). The well characterized Pfu Cmr (Type III-B) effector complex (Cmr1-6) interacts with two small crRNA species that are 45 and 39 nt in length, differing at their $3^{\prime}$ ends, and cleaves complementary target RNAs (Hale et al. 2009, 2012, 2014; Spilman et al. 2013; Ramia et al. 2014). The crRNA species that guide the function of the Type I Csa and Cst complexes in $P f u$ have not been delineated.

In this work, we have isolated and characterized two distinct native complexes comprised of the Csa (Type I-A) or Cst (Type I-G) Cas proteins and associated crRNAs originating from all seven Pfu CRISPR loci. These crRNAs associated with the Csa and Cst complexes have common $5^{\prime}$ ends generated by Cas6 cleavage (8-nt $5^{\prime}$ tag element) but possess different processed $3^{\prime}$ ends that often include a portion of the downstream repeat. We propose that these two newly identified Type I crRNPs, Csa (Type IA) and Cst (Type I-G), both function in crRNA-guided DNA cleavage of invading mobile genetic elements.

\section{RESULTS}

\section{Isolation of native Csa- and Cst- containing crRNP complexes}

Our previous biochemical fractionation analyses of $P$. furiosus (Pfu) cell extract revealed that crRNAs are components of chromatographically distinct RNAprotein complexes (Hale et al. 2008). One of the mature crRNA-containing 
complexes (crRNPs) was isolated and identified as the Type III-B (Cmr) complex that contains one of two major crRNA size forms (45 and $39 \mathrm{nt}$ ) and cleaves complementary RNA target (Hale et al. 2009, 2012, 2014; Spilman et al. 2013; Ramia et al. 2014). The other crRNPs identified by chromatography remained uncharacterized. In this work, we investigated the molecular composition of these uncharacterized effector complexes (crRNPs).

To gain insight into complexes containing Csa and Cst proteins, polyclonal antibodies were raised against Csa (Csa2 -a Cas7 superfamily protein, Cas3" Cas3 subunit with predicted effector nuclease activity) and Cst (Cst2 and Cas5t -Cas7 and Cas5 superfamily proteins, respectively) proteins (Fig. 1A). The antibody generated against $\mathrm{Cmr} 2$ was used as a positive control to isolate the previously characterized Cmr crRNP by this method. Preimmune (PI) antibodies (isolated before antigen injection) served as specificity controls. Csa, Cst, and Cmr polyclonal antibodies were first validated by Western blotting with Pfu cellular extract (Fig. 1B). All five antibodies recognized a protein of the expected size in Western blots confirming expression of each of these Cas proteins in Pfu.

\section{Cas protein composition of the isolated complexes}

We used these antibodies to immunopurify Csa-, Cst-, and Cmr-containing complexes from $P f u$ cell extract (Fig. 2). The protein components of the immunopurified complexes were identified by tandem mass spectrometry of tryptic peptides obtained from bands excised from silver-stained gels or shotgun analysis of samples eluted from the immunobeads (Fig. 2A,B; Supplemental Table 1; Supplemental Fig. S1). Mock immunoprecipitations, performed with matching preimmune antibodies, were used to filter for proteins specifically recognized by immune antibodies. As expected, Cmr2 antibodies selectively co-immunoprecipitated each of the six Cmr proteins (Cmr1-6) that were previously identified in the Cmr complex isolated through successive column chromatography (Hale et al. 2009, 2012). Immunopurifications performed with antibodies against two Csa (Type I-A) proteins (Csa2 [Cas7] and Cas3" [HD nuclease]) (see Fig. 1A), re-

B
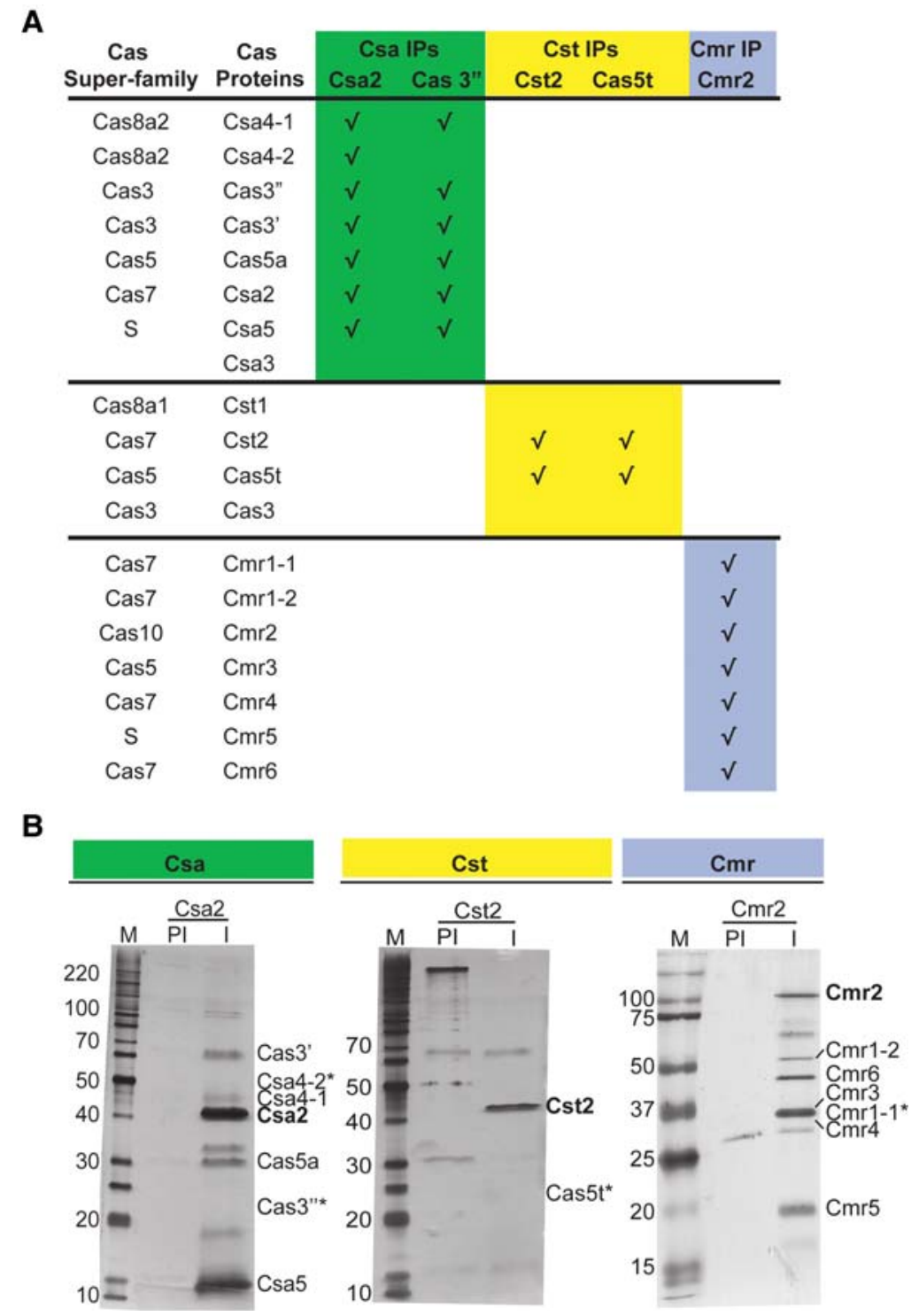

FIGURE 2. Cas protein composition of immunopurified Csa, Cst, and Cmr complexes. $(A)$ Cas proteins identified by tandem mass spectrometric analysis of Csa (green), Cst (yellow), Cmr (blue) immunoprecipitated complexes are indicated by a check mark. Some Cas proteins predicted to be part of a complex but not identified by mass spectrometry are also listed. $(B)$ Silverstained SDS-polyacrylamide gels of Csa (Csa2), Cst (Cst2), and Cmr (Cmr2) immunoprecipitated pellets with preimmune (PI) or immune (I) antibodies as labeled. Cas proteins identified by tandem mass spectrometry are indicated based on expected molecular weight. The antigens are labeled in bold. Cas proteins identified by mass spectrometry that do not correlate to a visible
band are marked with an asterisk. $\mathrm{M}$ is the protein standard in kilodaltons (kDa).

sulted in selective co-immunoprecipitation of each of the Csa proteins encoded in cas gene cluster 2 (Fig. 2; Supplemental Table 1; Supplemental Fig. S1) with the exception of the Csa3 protein (whose gene is configured in the opposite orientation to the rest of the genes in the csa operon) (Fig. 1A). Likewise, the two antibodies against the Cst proteins (Cst2 (Cas7) and Cas5t) selectively immunopurified only Cst proteins adjacently encoded in gene cluster 1 (Fig. 2; Supplemental Table 1; Supplemental Fig. S1). In this case, either antibody isolated both Cst 2 and Cas5t proteins while two 
other predicted components of the Cst (Type I-G) immune system (Cst1 [Cas8a1] and Cas3; see Fig. 1A), were not identified. A few non-Cas proteins were specifically detected in some of these immunopurifications (Supplemental Table 1).

To verify results of our mass spectrometry analyses, Western blotting was carried out for all five immunoprecipitation reactions (Csa2, Cas3", Cst2, Cas5t, and Cmr2) with each blot being probed with one of the antibodies (Fig. 3). The untreated $P f u$ cellular extract was used as a control in each blot to detect the antigen of expected molecular weight as in Figure 1B. As expected from the mass spectrometry results, Csa2 and Cas3" were both detected in complexes co-immunoprecipitated with Csa (Csa2, Cas3") antibodies. Cst2 and Cas5t were seen specifically in complexes isolated with Cst (Cst2 and Cas5t) antibodies, and Cmr2 was detected only in the Cmr complex. In summary, the findings (Figs. 2, 3; Supplemental Table 1; Supplemental Fig. S1) provide evi-
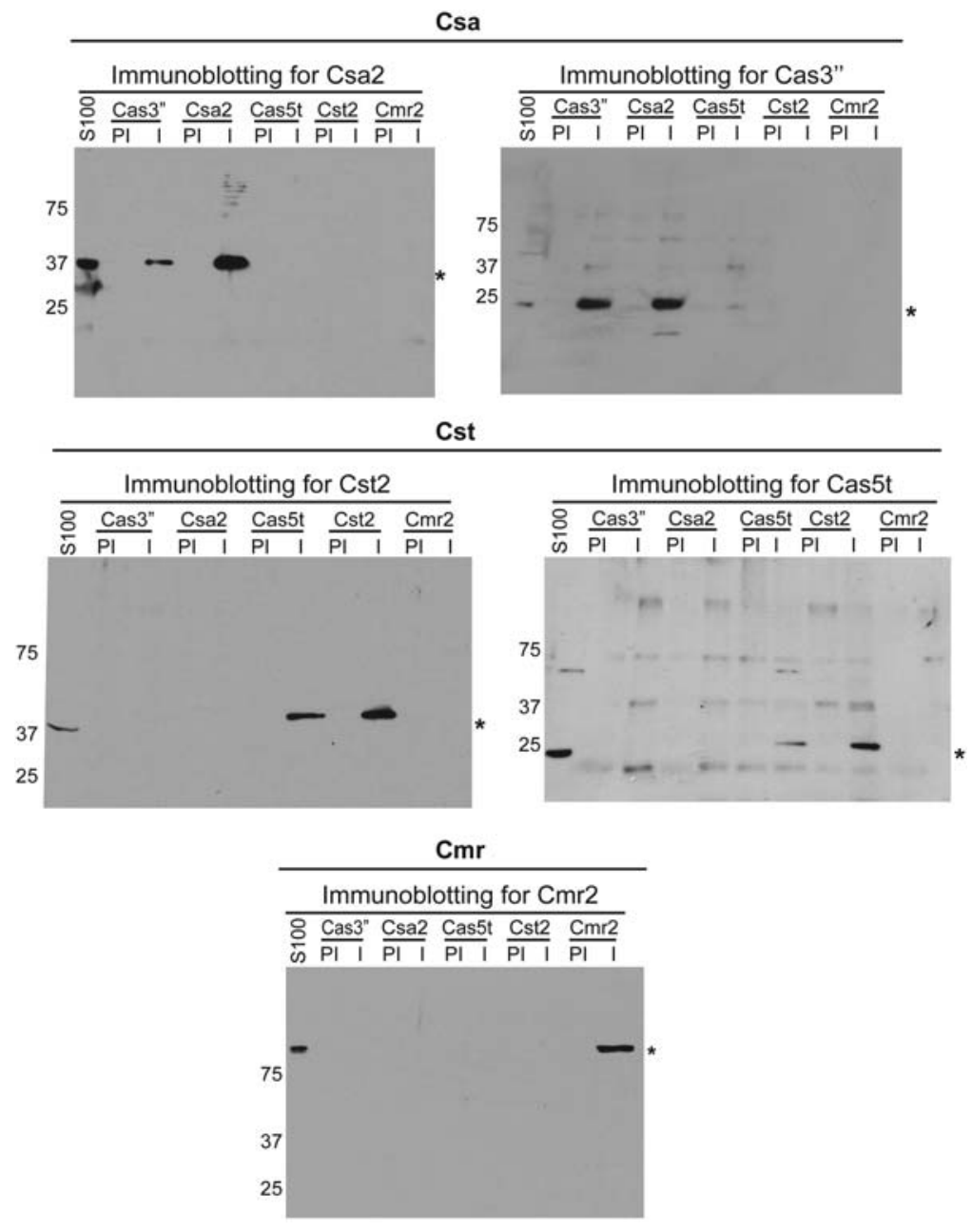

FIGURE 3. Western blot analysis of Cas proteins associated with Csa, Cst, and Cmr complexes. All five immunopurified samples were blotted and probed with each of the five immune antibodies (Csa2, Cas3", Cst2, Cas5t, Cmr2) individually. The first lane of each blot is untreated Pfu S100 extract used as a reference. An asterisk indicates the expected position of antigens based on their molecular weights. Molecular weights are indicated in kilodaltons $(\mathrm{kDa})$. dence for three compositionally distinct, $P f u$ complexes containing Csa, Cst, or Cmr proteins.

\section{Northern analysis of crRNAs associated with the Csa and Cst complexes}

To determine if the isolated Csa and Cst complexes also contained crRNAs, we performed Northern blot analysis (Fig. 4). RNAs extracted from the total RNA samples as well as the immunopurified complexes were probed for the first crRNA in CRISPR locus 7 (7.01 crRNA). The immunopurified $\mathrm{Cmr}$ complex shows the expected doublet of mature crRNAs of two major size forms (45 and $39 \mathrm{nt}$ ) (Hale et al. 2009, 2012). In contrast, the immunopurified Csa (Csa2, Cas3" immunoprecipitations) and Cst (Cst2, Cas5t immunoprecipitations) complexes show a profile that consists of more diverse crRNAs size forms ranging from 45 to $\sim 65 \mathrm{nt}$ in length with the 45-nt size form being the most prominent species in each sample. The prominent 39-nt crRNA band found in the total RNA and Cmr complex samples is not strongly associated with the Csa or Cst complexes.

\section{RNA sequencing of the associated crRNAs}

To gain a comprehensive understanding of the sequence features of crRNAs associated with Csa and Cst complexes, we sequenced both the total RNAs and complex-associated RNAs. crRNAs associated with immunopurified Csa (Csa2, Cas3"), Cst (Cst2, Cas5t), and Cmr (Cmr2) complexes map to all seven Pfu CRISPR loci (Fig. 5; Supplemental Figs. S2-S7) revealing that each expressed crRNA becomes integrated into three distinct CRISPR-Cas complexes. Figure 5 shows a representative profile of crRNAs from CRISPR locus 5 that associate with the Csa, Cst, and Cmr complexes compared with those in the Pfu cell extract (total RNA). As previously reported, the vast majority of crRNA reads in total RNA are on the sense strand (transcribed from promoters in leader sequences), and crRNA expression is generally higher at the leader-proximal end of the CRISPR locus (Fig. 5; Hale et al. 2008, 2012). These same trends hold true for crRNAs associated with the Csa, Cst, and Cmr complexes (Fig. 5). The profiles of individual crRNA reads associated with Csa and Cst complexes look similar to the 


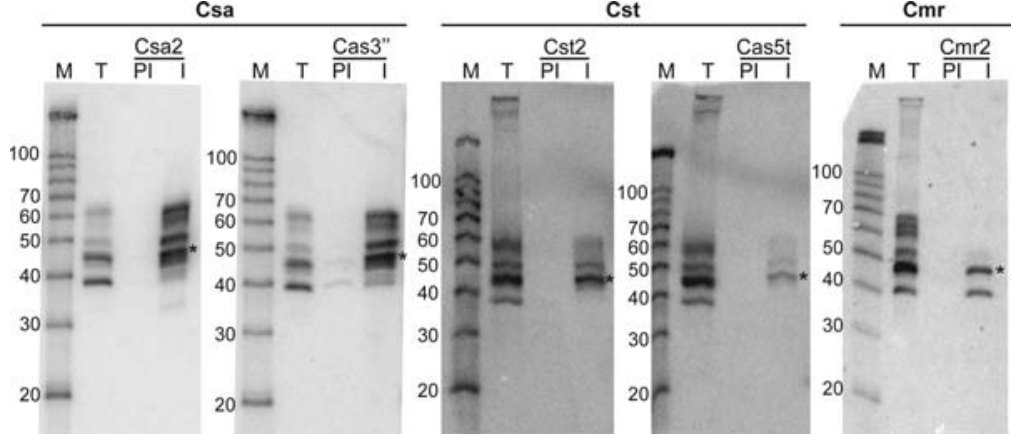

FIGURE 4. Northern blot analysis of crRNAs associated with Csa, Cst, and Cmr complexes. Northern blotting of RNAs extracted from $P f u$ S100 extract (T), preimmune pellets (PI), and immune pellets (I) of Csa (Csa2, Cas3"), Cst (Cst2, Cas5t), and Cmr (Cmr2). The blots were probed for the first crRNA of locus 7 (7.01). Asterisk indicates the prominent 45-nt crRNA band detected in all three complexes. Radiolabeled decade marker in nucleotides (M). plex blots probed for 7.01 detect longer crRNA species, which would correspond to +5 and +14 peaks seen in the sequencing data.

In summary, Csa, Cst, and Cmr complexes associated with a subset of processed crRNAs transcribed from all seven CRISPR loci. All complex-associated crRNAs harbor a $5^{\prime}$ tag sequence and appear to include one species of crRNA, ending at the guide- $3^{\prime}$ repeat junction. In general, the Csa- and Cst-associated crRNA forms share more similarity with each other than the Cmr-associated crRNAs. total crRNA profile and differ from Cmr-associated crRNAs, which show very sharp $3^{\prime}$ end boundaries. Similar crRNA expression trends are observed across all seven CRISPR loci (Supplemental Figs. S2-S7 show the profiles for the other CRISPR loci).

To map the $5^{\prime}$ and $3^{\prime}$ ends of crRNAs associated with immunopurified Csa (Csa2, Cas3" immunoprecipitations), Cst (Cst2, Cas5t immunoprecipitations), and $\mathrm{Cmr}$ (Cmr2 immunoprecipitation) complexes compared with the crRNA population in total RNA, we plotted $5^{\prime}$ and $3^{\prime}$ ends of crRNAs from RNA sequencing results (Fig. 6). The $5^{\prime}$ ends of the vast majority of crRNAs from the total crRNA samples as well as the complex-associated crRNAs contain the 8-nt $(-8)$ repeat-derived $5^{\prime}$-tag (AUUGAAAG), generated by Cas6 cleavage of pre-crRNA transcripts (Carte et al. 2008). In contrast, the $3^{\prime}$ end of the crRNAs associated with different complexes shows considerable heterogeneity. The crRNAs associated with Cmr complex exhibit two distinct peaks at the $3^{\prime}$ end, one ending at the guide-repeat junction $(-1)$ and the other peak $6 \mathrm{nt}$ upstream $(-7)$ and correspond to the previously characterized 45- and 39-nt size forms of Cmr complex-associated crRNAs, respectively (Hale et al. 2008, 2009, 2012). The crRNAs associated with the Csa (Csa2, Cas3") and Cst (Cst2, Cas5t) complexes exhibit heterogeneity at the $3^{\prime}$ end, containing variable amounts of $3^{\prime}$ repeat sequences. Both Csa and Cst associated crRNAs show one peak at the guide-repeat junction $(-1)$. There are other peaks in the downstream $3^{\prime}$ repeat $(+5,+14)$, corresponding to bigger crRNA forms. Another peak corresponds to a smaller crRNA form, with its $3^{\prime}$ extremity at $3 \mathrm{nt}$ upstream (Csa) and $4 \mathrm{nt}$ upstream (Cst) of the guide-repeat junction. Overall, the crRNA sizes obtained from mapping $5^{\prime}$ and $3^{\prime}$ ends (Fig. 6) are in accord with the crRNA species detected in Csa, Cst, and Cmr complexes by Northern blotting (Fig. 4). Northern blotting showed a 45-nt 7.01 crRNA in all three complexes, which would correspond to the -1 species at the $3^{\prime}$ guide-repeat junction. The 39-nt crRNA ( -7 form) specifically associates with the $\mathrm{Cmr}$ complex. The Csa, Cst com-

\section{DISCUSSION}

\section{Three distinct crRNPs coexist in Pyrococcus furiosus}

The $P f u$ genome encodes three potential CRISPR-Cas immune effector systems, Type I-A (Csa), Type I-G (Cst), and Type III-B (Cmr) systems (Fig. 1A). Here, we provide direct evidence that all three systems are expressed and assemble into compositionally distinct crRNPs. We hypothesize that each of these three coexisting crRNPs (Csa, Cst, and Cmr) can independently act as immune effector complexes that collectively mediate invader interference at both the RNA (Cmr) and DNA (Csa and Cst) levels (Fig. 7).

\section{Distinct profiles of crRNA size forms in Csa, Cst, and $\mathrm{Cmr}$ complexes}

Detailed analysis of the sizes of the crRNAs specifically associated with each of the Csa, Cst, and Cmr crRNP revealed that different forms of each crRNA are present in the three distinct complexes (Figs. 4, 6). The crRNAs have a common $5^{\prime}$ end (CRISPR repeat-derived 8-nt $5^{\prime}$ tag generated by Cas6 cleavage of primary CRISPR transcripts) but differ at their $3^{\prime}$ termini. While two major size forms of each crRNA (39 and $45 \mathrm{nt}$ in length) are associated with the Cmr complex, a broader collection of crRNA size forms containing various numbers of $3^{\prime}$ CRISPR repeat nucleotides occur in both the Csa and Cst complexes (Figs. 4, 6). A crRNA size form common in all three complexes in relatively high levels, the 45-nt crRNA species, contains the entire guide sequence and no $3^{\prime}$ repeat sequence (Figs. 4, 6). (The average encoded crRNA guide sequence [invader-derived] is $37 \mathrm{nt}$ in $P f u$.) In the case of Cmr-associated crRNAs, both the 45- and 39-nt forms are functional, suggesting that the precise $3^{\prime}$ terminus is not critical for activity (Hale et al. 2009, 2012). Structural studies reveal that each Cmr crRNP accommodates a single crRNA species (Zhang et al. 2012; Spilman et al. 2013; Staals et al. 2013; Ramia et al. 2014), and this is 


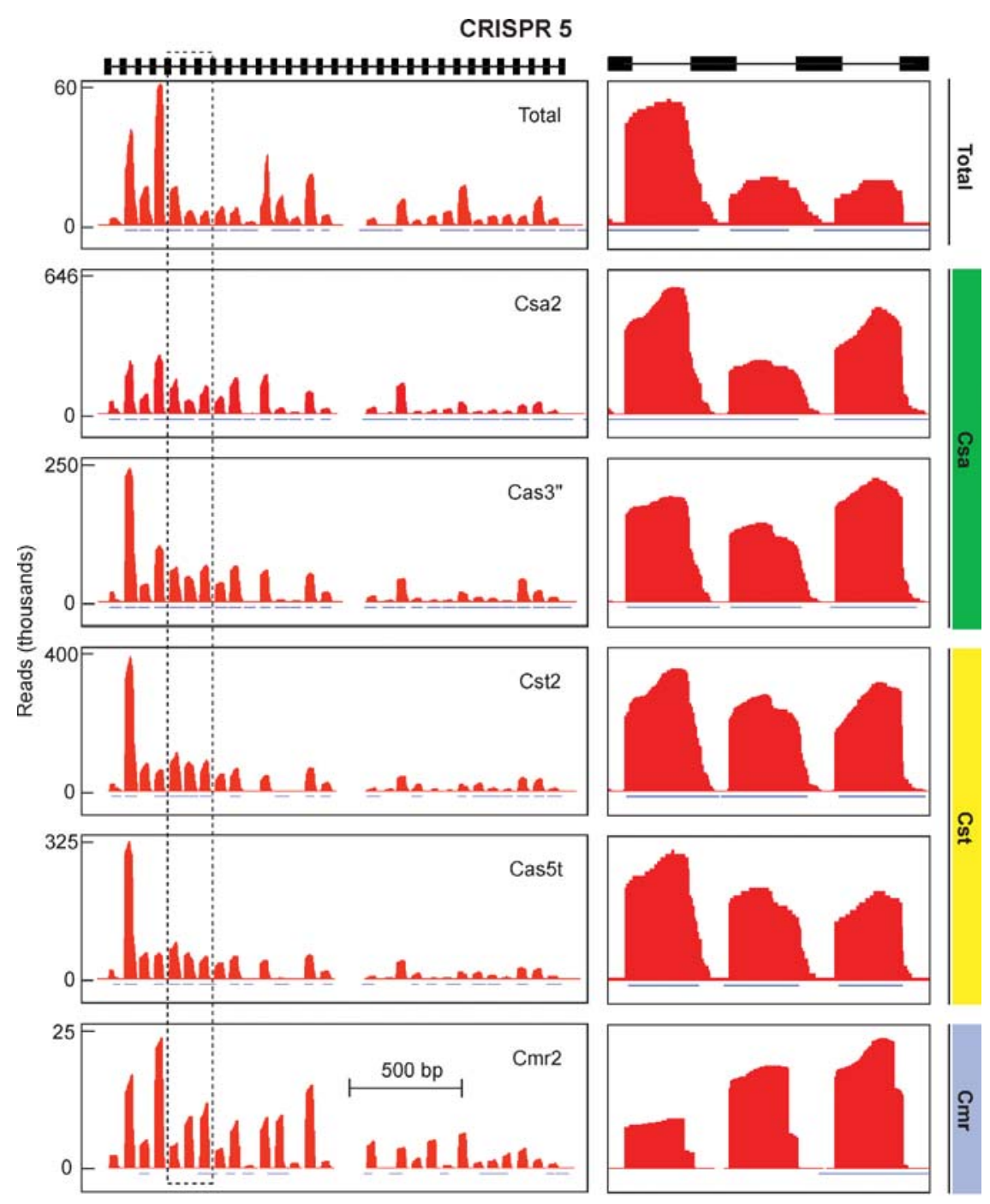

FIGURE 5. RNA-seq profiles of crRNAs associated with the Csa, Cst, and Cmr complexes and in total RNA. The number of reads (in thousands) of crRNAs-associated with each complex (extracted from immune pellets of Cas3", Csa2, Cas5t, Cst2, Cmr2) compared with crRNAs in total RNA (extracted from untreated S100 extract) that map to CRISPR locus 5 are shown. A higher resolution image of three crRNAs is highlighted in the box on the right. Reads mapping to both the sense (red, transcribed from upstream leader) and antisense (blue) strand are indicated. The black boxes at the top correspond to CRISPR repeats interspersed by guide sequences shown as black lines.

very likely the case for the Csa and Cst complexes based on recent structural information from other Type I systems (Jackson et al. 2014; Mulepati et al. 2014; Zhao et al. 2014). Further experiments are required to determine if all or a subset of the multiple size forms of crRNAs within the Csa and Cst crRNPs are functional or if crRNA $3^{\prime}$ trimming leads to functional activation for these specific complexes.

Molecular mechanisms governing crRNA $3^{\prime}$ end trimming in $P f u$ are unknown, but work from other systems suggests that non-Cas ribonucleases likely execute the $3^{\prime}$ trimming (Hatoum-Aslan et al. 2013). This finding suggests that the reason why each of the three crRNP complexes harbor crRNAs with distinct profiles of $3^{\prime}$ termini relates to differences in pro- tein protection afforded by each assembled Cas complex against further $3^{\prime}$ trimming into the body of the crRNA (Hatoum-Aslan et al. 2011, 2013). These results further indicate that $3^{\prime}$ end trimming most likely occurs coincident with or after Cas protein assembly.

\section{The Csa, Cst, and Cmr CRISPR-Cas complexes in $P f u$ incorporate crRNAs from seven CRISPR loci}

Unlike other CRISPR-Cas systems served by a single, integrated CRISPR array, the three CRISPR-Cas complexes found in $P f u$ utilize crRNAs from seven CRISPR loci distributed throughout the genome (including sites distal from the two cas gene clusters) (Terns and Terns 2013). While the complexes do not share a common identified protein component, the Cas6 endoribonuclease generates the crRNAs from all seven CRISPRs. Cas6 cleaves the primary CRISPR transcripts into $1 \mathrm{X}$ intermediate RNAs that contain the crRNA guide region flanked by $5^{\prime}$ and $3^{\prime}$ repeat sequences (8-nt repeat at $5^{\prime}$ end $\left[5^{\prime} \mathrm{tag}\right]$ and $22-\mathrm{nt}$ repeat at $3^{\prime}$ end) (Fig. 7; Carte et al. 2008; Hale et al. 2008, 2012). Moreover, our previous immunoprecipitation analysis indicates that Cas6 stably associates with $1 \mathrm{X}$ intermediate crRNAs in vivo (though not with $3^{\prime}$ trimmed crRNAs wherein the Cas6 binding sequence is lost) (Carte et al. 2010; Wang et al. 2011). Cas6 was not observed by mass spectrometry to be a component of isolated Csa, Cst, or Cmr crRNPs (Fig. 2; Supplemental Table 1). Taken together, the data indicate that Cas6 dissociates before or during crRNP assembly and crRNA $3^{\prime}$ end trimming, and thus that Cas6 may play a transient role in directly or indirectly incorporating crRNAs into the three distinct crRNPs (Carte et al. 2008).

The $5^{\prime}$ tag sequence likely plays a critical role in nucleating crRNA assembly into each of the three complexes. The 8-nt $5^{\prime}$ tag sequence is the only common sequence element of the 200 or so crRNAs in the total RNA pool and is critical for Cmr crRNP assembly and function (Hale et al. 2008, 2012, 2014; Zhang et al. 2012). While the protein components of the Csa, Cst, and Cmr complexes are distinct, all three complexes contain Cas 5 superfamily proteins. Recent work supports a role for Cas 5 superfamily proteins in mediating direct contact with $5^{\prime}$ tag sequences of crRNAs (Spilman et al. 2013; 

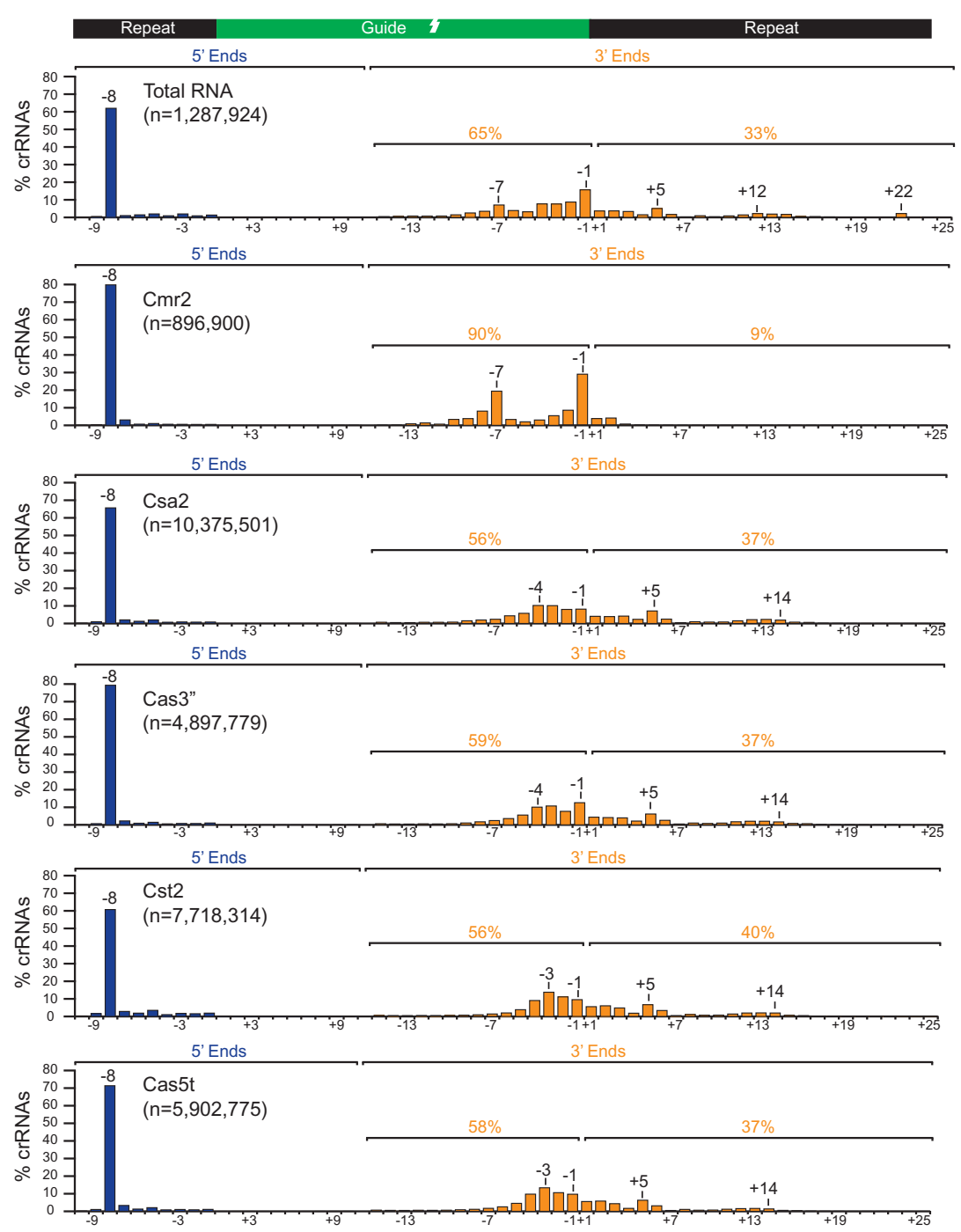

FIGURE 6. Mapping ends of crRNAs in total RNA and crRNAs associated with Csa, Cst, Cmr complexes. $5^{\prime}$ and $3^{\prime}$ end analysis of all sequenced crRNAs in untreated S100 extract (total RNA) and crRNAs associated with Csa (Csa2, Cas3"), Cst (Cst2, Cas5t), and Cmr (Cmr2) complexes. The percentage of crRNAs starting and ending at a specific point is marked on the $y$-axis. The nucleotides are counted relative to their position from the guide-repeat junction. The nucleotides upstream of the guide-repeat junction are counted from -1 and those downstream are counted from +1 . The peaks in the $5^{\prime}$ repeat region are shown in blue. The peaks downstream from the $5^{\prime}$ repeat-guide junction are shown in orange. The schematic on top indicates where the peaks map (repeats black and guides green).

Jackson et al. 2014; Mulepati et al. 2014; Zhao et al. 2014), including in the $\mathrm{Cmr}$ complex where the $\mathrm{Cmr} 3$ protein is implicated in direct $5^{\prime}$ tag binding (Spilman et al. 2013; Ramia et al. 2014). Presumably, the Cas5 superfamily proteins of the Csa and Cst complex (Cas5a and Cas5t, respectively) recognize the $5^{\prime}$ tag sequences in the context of Csa and Cst crRNPs. Interestingly, our data suggest that these Cas5 proteins may recognize slightly different features of these $5^{\prime}$ tag sequences. For example, the first 10 crRNAs from CRISPR eight are underrepresented in $\mathrm{Cmr}$ complexes as we have reported earlier (Hale et al. 2012), but not in Csa and Cst complexes (Supplemental Fig. S7). These crRNAs have a single nucleotide variation in the repeat-derived $5^{\prime}$ tag sequence that apparently accounts for their ability to associate with the Csa and Cst complex but not the Cmr complex.

\section{Cas protein composition and functional roles of Csa and Cst crRNPs}

Our data strongly support the existence of three separate crRNPs each containing non-overlapping Cas protein subunits (Figs. 2, 3; Supplemental Table 1; Supplemental Fig. S1). The immunopurified Type III-B Cmr complexes contain all $\mathrm{Cmr}$ proteins (Cmr1-6) that have been previously identified by conventional column chromatography purification (Hale et al. 2009) and shown to be individually required for $\mathrm{Cmr}$ RNA targeting activity in vitro using recombinant proteins (Hale et al. 2009, 2012, 2014). DNA targeting in addition to RNA targeting activity has been found for the Cmr complex in Sulfolobus islandicus (Deng et al. 2013; Peng et al. 2015). Similar to the $\mathrm{Cmr}$ complex, immunopurified Type I-A (Csa) crRNPs were found to contain each of the encoded Csa proteins with the exception of $\mathrm{Csa} 3$, a protein thought to be a transcriptional regulator of csa genes rather than a component of the Csa effector complex (Lintner et al. 2011a; Makarova et al. 2014). The first experimental evidence for the existence of native Csa crRNPs came from work with the thermophilic archaeaon Sulfolobus solfataricus, where affinity purifications performed with tagged Csa2 (Cas7) provided evidence for a stable interaction of Csa2 (Cas7) with Cas5a (Cas5) and crRNA with possible additional (less stable) interactions with Csa5 (Cas11 or small subunit protein), Csa4 (Cas8), and Cas6 (Lintner et al. 2011b). Similar to our findings, Type I-A (Csa) crRNPs reconstituted with recombinant proteins in Thermoproteus tenax contained Cas3" and Cas3' as stable components of the complex (Plagens et al. 2012, 2014). The recombinant $T$. tenax Csa crRNP targets DNA, and Cas3" (HD-nuclease protein) is the effector nuclease (Plagens et al. 2014). We expect the stably associated Cas3" is likely the effector nuclease of the predicted DNA-targeting Type I-A (Csa) effector complex in $P f u$.

The predicted Cas proteins required for a functional Type I-G (Cst) crRNP include Cst1, Cst2, Cas5t, and Cas3 (Haft 


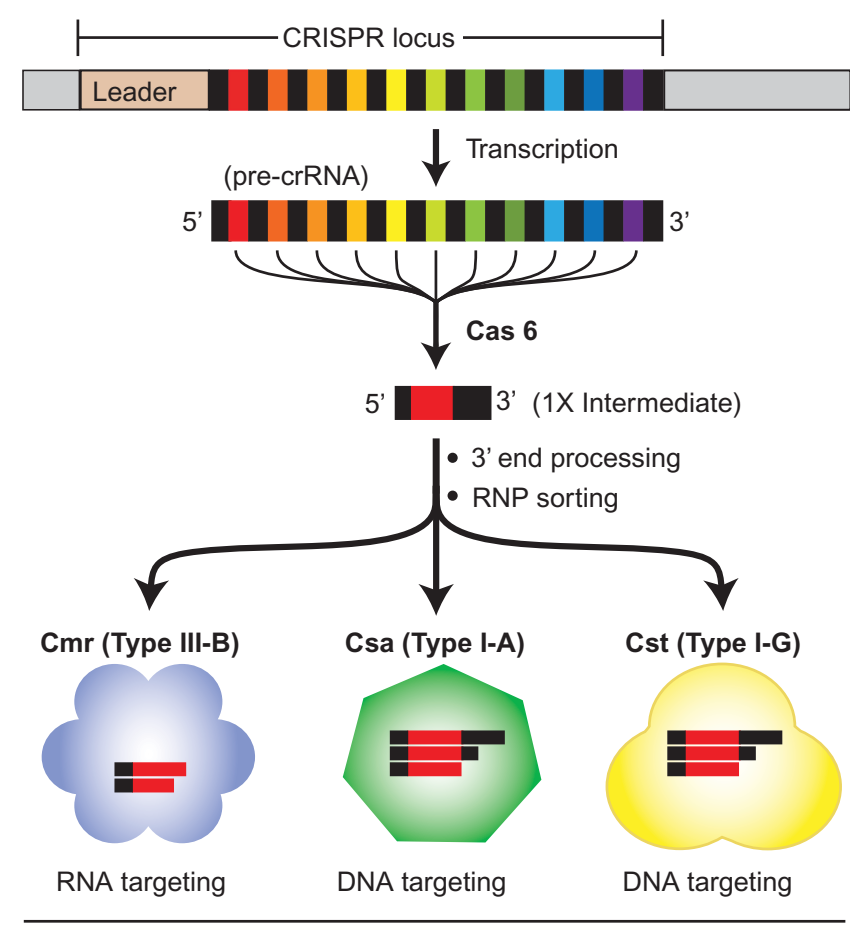

Effector crRNPs

FIGURE 7. Model for integration of crRNAs into three distinct crRNP effector complexes. The CRISPR locus (repeat: black rectangles, invader-derived guide sequences: multiple colors) is transcribed from a promoter in the leader (brown) to form the pre-crRNA. Cas6 cleaves once in each repeat element of the pre-cRNA transcript to generate 1X RNA intermediates that each contain the $5^{\prime}$ tag, entire guide sequence, and 22 nt of $3^{\prime}$ repeat. Further $3^{\prime}$ end trimming by unknown mechanisms occurs either before, during, or after association with specific Cas proteins. The Cmr complex (blue) associates with two forms of crRNAs lacking any $3^{\prime}$ repeat. Csa (green) and Cst (yellow) complexes harbor crRNAs that contain variable amounts of $3^{\prime}$ repeat and one crRNA species without any $3^{\prime}$ repeat. Each crRNA size form can assemble into the corresponding effector complexes (one crRNA/complex) and guide targeting. The Cmr complex cleaves complementary RNA targets. The Csa and Cst effector complexes are predicted to target DNA homologous to the guide sequences in the crRNAs.

et al. 2005; Makarova et al. 2011; Vestergaard et al. 2014). Our immunopurified complexes contained Cst 2 and Cas $5 \mathrm{t}$ but lacked Cst 1 and Cas3. Cst2 and Cas5t are Cas7 and Cas5 superfamily proteins of Type I-G systems that likely make up the stable crRNA-binding core of the complex. Analysis of Type I-E (Cse) crRNPs provided support that the large subunit protein Cse1 (Cas8 superfamily) is a relatively weakly associated and peripheral component of the complex (Jore et al. 2011). Likewise, Cst1 (also a Cas8 superfamily protein) may be less stably associated with the Type I-G (Cst) crRNP. While we failed to identify the Cst 1 subunit by our immunopurification approach (Fig. 2), Pfu Cst1 was found in complex with both Cst 2 and Cas5t following sequential nondenaturing column chromatography fractionations (Menon et al. 2009). Collectively, the data suggest that all three Cst proteins (Cst1, Cst2, Cas5t) are integral subunits of Type I-G crRNPs. We predict that Cas3 (PF1120), which occurs immediately adjacent to cst genes in the gene cluster (Fig. 1A) will cofunction with the Cst complex as the effector nuclease/helicase. Based on expectations from other Type I systems, the Cst crRNP may function as a surveillance complex that recruits Cas3 nuclease to cleave invader DNA (Brouns et al. 2008; Cady and O’Toole 2011; Jore et al. 2011; Wiedenheft et al. 2011a,b; Nam et al. 2012; Sashital et al. 2012; van Duijn et al. 2012; Westra et al. 2012b; Mulepati and Bailey 2013; Sinkunas et al. 2013; Hochstrasser et al. 2014; Huo et al. 2014). We likely did not detect Cas3 in our isolated Cst crRNP complexes (Fig. 2) because the Cas3 protein may not associate with Cst complexes in the absence of (invader) DNA targets (Hochstrasser et al. 2014; Huo et al. 2014).

A small number of non-Cas proteins were identified in the Csa, Cst, and/or Cmr complex, but further work is required to understand if any of these proteins are functionally significant for crRNP regulation or function (Supplemental Table 1). For example, the thermosome subunit (PF1974), identified in Csa immunoprecipitations, is a heat shock protein (Hsp60 homolog) and could act as a chaperone in proper folding of some Cas proteins (Klumpp and Baumeister 1998; Shockley et al. 2003). By analogy, previous work suggests that an Escherichia coli chaperonin protein (high temperature protein $\mathrm{G}$ ) is essential for maintaining functional levels of Cas3 and thereby CRISPR-mediated silencing (Yosef et al. 2011). Moreover, PF1563, a subunit of DNA-dependent RNA polymerase was also identified in Csa immunoprecipitations. Interestingly, Thermococcus kodakarensis Csa2 (TK0462) was also shown to copurify with RNA Polymerase (Santangelo et al. 2007) indicating a potential conserved interaction between Csa components and transcriptional machinery.

\section{Advantages of multiple CRISPR-Cas systems}

While some organisms have a single CRISPR-Cas immune system, many harbor more than one system. The three coexisting complexes of $P f u$ very likely target invaders at both RNA (Cmr) and DNA (Csa, Cst) levels for silencing (Fig. 7). Having multiple effector complexes offers potential advantages. We now know that crRNAs from all seven loci associate with each of the three (Csa, Cst, Cmr) coexisting complexes in Pfu (Figs. 4, 5; Supplemental Figs. 2-7). Therefore, a single crRNA can potentially guide targeting by each of the three complexes. Having both RNA- and DNA-targeting systems could provide silencing of different types of viruses and other mobile genetic elements that for $P f u$ remain largely uncharacterized. Furthermore, we speculate that having multiple, and potentially redundant, CRISPR-Cas immune systems would be highly beneficial in the event that a virally encoded "anti-CRISPR" gene functions to inactivate a specific CRISPR-Cas type as has been observed for phages that infect Pseudomonas aeruginosa (Bondy-Denomy et al. 2013; Pawluk et al. 2014). The identification of the Csa and Cst crRNPs described in this study lays the foundation for a 
more detailed structural and functional analysis of these complexes akin to those that have been performed to understand the previously identified Cmr complex (Hale et al. 2009, 2012, 2014; Spilman et al. 2013; Ramia et al. 2014). Further investigation of these three potentially independent and cofunctioning complexes will provide insights into multiple layers of CRISPR-Cas mediated invader defense in P. furiosus.

\section{MATERIALS AND METHODS}

\section{Preparation of polyclonal antibodies against Cas proteins}

For antigen production, csa2, cas3" (pET24D, Novagen), cas5t, cst2, and cmr2 (pET200D, Invitrogen) genes were cloned and transformed into E. coli (BL21 RIPL cells, Agilent Technologies). $\mathrm{N}$-terminal, 6X His-tagged recombinant proteins were expressed in E. coli by inducing with $1 \mathrm{mM}$ IPTG at mid-log growth phase $\left(\mathrm{OD}_{600}\right.$ at $\left.0.4-0.7\right)$ and incubated overnight at room temperature with shaking and purified by nickel chromatography as described (Hale et al. 2009). Polyclonal antibodies against each antigen were raised in chickens and purified as previously described (Carte et al. 2010).

\section{Preparation of Pfu cell extract}

Approximately $5 \mathrm{~g}$ of $P f u$ cells (DSM3638) were resuspended in 20 $\mathrm{mL}$ of $50 \mathrm{mM}$ Tris ( $\mathrm{pH} 8.0)$ in the presence of 100 units of RQ1 DNase (Promega) and $0.1 \mathrm{mM}$ phenylmethanesulfonylfluoride (PMSF) and lysed by stirring at room temperature. The cell extract was then centrifuged at $100,000 \mathrm{~g}$ for $90 \mathrm{~min}$ at $4^{\circ} \mathrm{C}$ using a SW41 Ti rotor (Beckman). The resulting S100 extract, with a typical total protein concentration of $20 \mathrm{mg} / \mathrm{mL}$, was stored at $-80^{\circ} \mathrm{C}$ until further use.

\section{Immunoprecipitation of Csa, Cst, and Cmr-containing complexes}

Immunoprecipitations were performed using anti-Csa2, anti-Cas3", anti-Cst2, anti-Cas5t, and anti-Cmr2 IgY antibodies conjugated either directly to CarboLink-coupled beads (Pierce) or indirectly coupled to anti-IgY-conjugated agarose beads (Gallus Immunotech). The coupling was performed as per manufacturer's recommended protocol and efficiency verified by recording A280 readings (before and after coupling reaction) and subsequent analysis via SDS-PAGE. Immunoprecipitation reactions with the direct conjugated method were used for mass spectrometry analysis of protein samples and those done with the indirect (IgY) coupling method were used for Northern analysis and RNA sequencing.

An individual immunoprecipitation reaction typically contained $270-550 \mu \mathrm{g}$ of the antibody-conjugated beads, $200 \mu \mathrm{L}$ of Pfu S100 extract ( $\sim 4 \mathrm{mg}$ of total protein), 1X Complete Mini protease inhibitor (Roche), and 50 units of SUPERase-IN RNase inhibitor (Applied Biosystems), brought up to a final volume of $1 \mathrm{~mL}$ with IPP 300 (10 mM Tris ( $\mathrm{pH} 8.0$ ), $300 \mathrm{mM} \mathrm{NaCl}, 0.05 \%$ Igepal). The reaction was then incubated at room temperature for $2 \mathrm{~h}$ with end-overend rotation followed by centrifugation at $3000 \mathrm{~g}$ for $2 \mathrm{~min}$. The su- pernatant was stored at $4^{\circ} \mathrm{C}$ for any further analysis (e.g., Western or Northern blotting). The pellets were washed four times in $1 \mathrm{~mL}$ of IPP 300 .

\section{Mass spectrometry sample preparation}

For in-solution mass spectrometry analysis, the immunoprecipitated samples (preimmune and immune) were washed 2-4 times in 1 $\mathrm{mL}$ of $1 \mathrm{X}$ PBS to remove excess detergent and eluted in $100 \mu \mathrm{L}$ of $100 \mathrm{mM}$ glycine at $\mathrm{pH} 2.8$ for $2-10 \mathrm{~min}$. The eluted material was quickly neutralized in $10 \mu \mathrm{L}$ of $1 \mathrm{M}$ sodium phosphate buffer $(\mathrm{pH}$ 8.8). For in-gel mass spectrometry analysis, the immunoprecipitated samples (preimmune and immune) were resuspended in SDS loading buffer (without reducing agent) and heated at $65^{\circ} \mathrm{C}$ for $5 \mathrm{~min}$ followed by electrophoretic separation on 12\% Mini-PROTEAN TGX gels (Biorad). The gels were either silver stained (Blum et al. 1987; Shevchenko et al. 1996) and bands were excised or unstained gel portions were excised.

\section{Protein assignment by tandem mass spectrometry}

The data generated comes from two to five separate immunoprecipitation replicates. In-gel and in-solution tryptic digests were performed essentially as described previously (Wells et al. 2002; Lim et al. 2008; Hale et al. 2009). The desalted tryptic peptides were analyzed by nanoESI-LC-MS/MS on a linear ion trap mass spectrometer (LTQ XL, Thermo Fisher Scientific) as described previously (Lim et al. 2008). The acquired data were searched against a Pyrococcus furiosus specific database (Uniprot, updated on December 21, 2009) using SEQUEST (Thermo Fisher Scientific). The search output was filtered to obtain a $1 \%$ protein false discovery rate ( $99 \%$ confidence) using the ProValT algorithm as deployed in PROTEOIQ (www .nusep.com). The proteins identified in Supplemental Table 1 were not present in the corresponding preimmune control samples and contained two or more peptides.

\section{Western blotting}

Pfu S100 extract ( $\sim 40 \mu$ g total protein) and one-third of an immunoprecipitation reaction were typically used. Western blotting was performed by standard procedures. The blots were incubated with 1:2500-1:100,000 dilution of polyclonal IgY immune antibodies and 1:5000 dilution of HRP-conjugated Anti-IgY secondary antibody (Gallus Immunotech). The protein bands on the blots were detected using an enhanced chemiluminescent substrate for HRP (horse radish peroxidase) activity (ECL, Thermo Scientific Pierce).

\section{Northern analysis}

Total RNAs were extracted from $P f u$ S100 extract and immunoprecipitated samples (preimmune and immune) using TRIzol LS Reagent (Invitrogen) as per the manufacturer's recommendation. Total RNA $(2 \mu \mathrm{g})$ from S100 extract and one-fourth (Csa2), onehalf (Cas5t, Cst2, Cmr2), and 2 reactions (Cas3") of immunoprecipitation samples were used. Northern blots were performed as described previously (Hale et al. 2008). Blots were probed with $5^{\prime}$ ${ }^{32}$ P-labeled crRNA 7.01 antisense oligo (Hale et al. 2008) and exposed on a phosphorimager screen overnight. 


\section{Illumina sequencing of crRNAs}

Total RNA (50 $\mu \mathrm{g})$ from Pfu S100 extract and Csa, Cst, and Cmr immunoprecipitation samples (immune) were extracted using TRIzol LS reagent (Life Technologies). RNAs were electrophoresed on a 10\% PAGE with $7 \mathrm{M}$ urea alongside oligonucleotide size standards, stained with SYBR-gold (Life Technologies), and gel slices in the size range of 19-70 nt were excised. The libraries were prepared as described (Hale et al. 2012). Briefly, the RNAs were phosphatase-treated to remove $3^{\prime}$ phosphates and ligated to an adaptor. These products were treated with T4 polynucleotide kinase before ligation of the $5^{\prime}$ end to another adaptor. The products were reverse transcribed, followed by PCR. The samples were diluted to a concentration of $24 \mathrm{pM}$ and subjected to 76 cycles of sequencing on an Illumina Genome Analyzer II. Reads were trimmed of the linker sequences and aligned to the genome using Bowtie (Langmead et al. 2009) and the end analysis performed as previously described (Hale et al. 2012).

\section{DATA DEPOSITION}

All RNA sequence data are available from GEO under accession numbers GSE33256 and GSE65781.

\section{SUPPLEMENTAL MATERIAL}

Supplemental material is available for this article.

\section{ACKNOWLEDGMENTS}

We thank Joshua Elmore for his contribution in preparing total $P$. furiosus RNA for RNA sequencing. We thank members of Terns' laboratory for scientific discussions and technical input including team-effort preparation of antibodies. This work was supported by a National Institutes of Health Grant R01 GM54682 to M.P.T. and R.M.T. and in part by the Georgia Research Alliance and the National Center for Biomedical Glycomics P41GM103490 to L.W., Hatch Funds to M.C., and grants R01 GM095296 and R01 GM067842 to B.R.G.

Received November 26, 2014; accepted February 25, 2015.

\section{REFERENCES}

Barrangou R, Fremaux C, Deveau H, Richards M, Boyaval P, Moineau S, Romero DA, Horvath P. 2007. CRISPR provides acquired resistance against viruses in prokaryotes. Science 315: 1709-1712.

Blum H, Beier H, Gross HJ. 1987. Improved silver staining of plant proteins, RNA and DNA in polyacrylamide gels. Electrophoresis 8: 93-99.

Bolotin A, Quinquis B, Sorokin A, Ehrlich SD. 2005. Clustered regularly interspaced short palindrome repeats (CRISPRs) have spacers of extrachromosomal origin. Microbiology 151: 2551-2561.

Bondy-Denomy J, Pawluk A, Maxwell KL, Davidson AR. 2013. Bacteriophage genes that inactivate the CRISPR/Cas bacterial immune system. Nature 493: 429-432.

Brendel J, Stoll B, Lange SJ, Sharma K, Lenz C, Stachler AE, Maier LK, Richter H, Nickel L, Schmitz RA, et al. 2014. A complex of Cas proteins 5,6 , and 7 is required for the biogenesis and stability of clustered regularly interspaced short palindromic repeats (CRISPR)derived RNAs (crRNAs) in Haloferax volcanii. J Biol Chem 289: 7164-7177.
Brouns SJ, Jore MM, Lundgren M, Westra ER, Slijkhuis RJ, Snijders AP, Dickman MJ, Makarova KS, Koonin EV, van der Oost J. 2008. Small CRISPR RNAs guide antiviral defense in prokaryotes. Science 321: 960-964.

Cady KC, O’Toole GA. 2011. Non-identity-mediated CRISPR-bacteriophage interaction mediated via the Csy and Cas3 proteins. J Bacteriol 193: 3433-3445.

Carte J, Wang R, Li H, Terns RM, Terns MP. 2008. Cas6 is an endoribonuclease that generates guide RNAs for invader defense in prokaryotes. Genes Dev 22: 3489-3496.

Carte J, Pfister NT, Compton MM, Terns RM, Terns MP. 2010. Binding and cleavage of CRISPR RNA by Cas6. RNA 16: 2181-2188.

Deng L, Garrett RA, Shah SA, Peng X, She Q. 2013. A novel interference mechanism by a type IIIB CRISPR-Cmr module in Sulfolobus. Mol Microbiol 87: 1088-1099.

Elmore JR, Yokooji Y, Sato T, Olson S, Glover CV III, Graveley BR, Atomi H, Terns RM, Terns MP. 2013. Programmable plasmid interference by the CRISPR-Cas system in Thermococcus kodakarensis. RNA Biol 10: 828-840.

Fischer S, Maier LK, Stoll B, Brendel J, Fischer E, Pfeiffer F, DyallSmith M, Marchfelder A. 2012. An archaeal immune system can detect multiple protospacer adjacent motifs (PAMs) to target invader DNA. J Biol Chem 287: 33351-33363.

Garneau JE, Dupuis MÈ, Villion M, Romero DA, Barrangou R, Boyaval P, Fremaux C, Horvath P, Magadán AH, Moineau S. 2010. The CRISPR/Cas bacterial immune system cleaves bacteriophage and plasmid DNA. Nature 468: 67-71.

Gasiunas G, Barrangou R, Horvath P, Siksnys V. 2012. Cas9-crRNA ribonucleoprotein complex mediates specific DNA cleavage for adaptive immunity in bacteria. Proc Natl Acad Sci 109: E2579-E2586.

Gasiunas G, Sinkunas T, Siksnys V. 2014. Molecular mechanisms of CRISPR-mediated microbial immunity. Cell Mol Life Sci 71: 449465.

Grissa I, Vergnaud G, Pourcel C. 2007. The CRISPRdb database and tools to display CRISPRs and to generate dictionaries of spacers and repeats. BMC Bioinformatics 8: 172 .

Haft DH, Selengut J, Mongodin EF, Nelson KE. 2005. A guild of 45 CRISPR-associated (Cas) protein families and multiple CRISPR/ Cas subtypes exist in prokaryotic genomes. PLoS Comput Biol 1: e60.

Hale C, Kleppe K, Terns RM, Terns MP. 2008. Prokaryotic silencing (psi)RNAs in Pyrococcus furiosus. RNA 14: 2572-2579.

Hale CR, Zhao P, Olson S, Duff MO, Graveley BR, Wells L, Terns RM, Terns MP. 2009. RNA-guided RNA cleavage by a CRISPR RNA-Cas protein complex. Cell 139: 945-956.

Hale CR, Majumdar S, Elmore J, Pfister N, Compton M, Olson S, Resch AM, Glover CV III, Graveley BR, Terns RM, et al. 2012. Essential features and rational design of CRISPR RNAs that function with the Cas RAMP module complex to cleave RNAs. Mol Cell 45: 292-302.

Hale CR, Cocozaki A, Li H, Terns RM, Terns MP. 2014. Target RNA capture and cleavage by the Cmr type III-B CRISPR-Cas effector complex. Genes Dev 28: 2432-2443.

Hatoum-Aslan A, Maniv I, Marraffini LA. 2011. Mature clustered, regularly interspaced, short palindromic repeats RNA (crRNA) length is measured by a ruler mechanism anchored at the precursor processing site. Proc Natl Acad Sci 108: 21218-21222.

Hatoum-Aslan A, Samai P, Maniv I, Jiang W, Marraffini LA. 2013. A ruler protein in a complex for antiviral defense determines the length of small interfering CRISPR RNAs. J Biol Chem 288: 27888-27897.

Hochstrasser ML, Taylor DW, Bhat P, Guegler CK, Sternberg SH, Nogales E, Doudna JA. 2014. CasA mediates Cas3-catalyzed target degradation during CRISPR RNA-guided interference. Proc Natl Acad Sci 111: 6618-6623.

Huo Y, Nam KH, Ding F, Lee H, Wu L, Xiao Y, Farchione MD Jr, Zhou S, Rajashankar K, Kurinov I, et al. 2014. Structures of CRISPR Cas3 offer mechanistic insights into Cascade-activated DNA unwinding and degradation. Nat Struct Mol Biol 21: 771-777.

Jackson RN, Golden SM, van Erp PB, Carter J, Westra ER, Brouns SJ, van der Oost J, Terwilliger TC, Read RJ, Wiedenheft B. 2014. 
Structural biology. Crystal structure of the CRISPR RNA-guided surveillance complex from Escherichia coli. Science 345: 1473-1479.

Jansen R, Embden JD, Gaastra W, Schouls LM. 2002. Identification of genes that are associated with DNA repeats in prokaryotes. Mol Microbiol 43: 1565-1575.

Jinek M, Chylinski K, Fonfara I, Hauer M, Doudna JA, Charpentier E. 2012. A programmable dual-RNA-guided DNA endonuclease in adaptive bacterial immunity. Science 337: 816-821.

Jore MM, Lundgren $M$, van Duijn E, Bultema JB, Westra ER, Waghmare SP, Wiedenheft B, Pul U, Wurm R, Wagner R, et al. 2011. Structural basis for CRISPR RNA-guided DNA recognition by Cascade. Nat Struct Mol Biol 18: 529-536.

Klumpp M, Baumeister W. 1998. The thermosome: archetype of group II chaperonins. FEBS Lett 430: 73-77.

Langmead B, Trapnell C, Pop M, Salzberg SL. 2009. Ultrafast and memory-efficient alignment of short DNA sequences to the human genome. Genome Biol 10: R25.

Lillestol RK, Redder P, Garrett RA, Brugger K. 2006. A putative viral defence mechanism in archaeal cells. Archaea 2: 59-72.

Lim JM, Sherling D, Teo CF, Hausman DB, Lin D, Wells L. 2008. Defining the regulated secreted proteome of rodent adipocytes upon the induction of insulin resistance. J Proteome Res 7: 12511263.

Lintner NG, Frankel KA, Tsutakawa SE, Alsbury DL, Copié V, Young MJ, Tainer JA, Lawrence CM. 2011a. The structure of the CRISPR-associated protein Csa3 provides insight into the regulation of the CRISPR/Cas system. J Mol Biol 405: 939-955.

Lintner NG, Kerou M, Brumfield SK, Graham S, Liu H, Naismith JH, Sdano M, Peng N, She Q, Copié V, et al. 2011b. Structural and functional characterization of an archaeal clustered regularly interspaced short palindromic repeat (CRISPR)-associated complex for antiviral defense (CASCADE). J Biol Chem 286: 21643-21656.

Maier LK, Lange SJ, Stoll B, Haas KA, Fischer S, Fischer E, DuchardtFerner E, Wöhnert J, Backofen R, Marchfelder A. 2013. Essential requirements for the detection and degradation of invaders by the Haloferax volcanii CRISPR/Cas system I-B. RNA Biol 10: 865-874.

Makarova KS, Grishin NV, Shabalina SA, Wolf YI, Koonin EV. 2006. A putative RNA-interference-based immune system in prokaryotes: computational analysis of the predicted enzymatic machinery, functional analogies with eukaryotic RNAi, and hypothetical mechanisms of action. Biol Direct 1: 7.

Makarova KS, Aravind L, Wolf YI, Koonin EV. 2011. Unification of Cas protein families and a simple scenario for the origin and evolution of CRISPR-Cas systems. Biol Direct 6: 38.

Makarova KS, Anantharaman V, Grishin NV, Koonin EV, Aravind L. 2014. CARF and WYL domains: ligand-binding regulators of prokaryotic defense systems. Front Genet 5: 102.

Marraffini LA, Sontheimer EJ. 2008. CRISPR interference limits horizontal gene transfer in staphylococci by targeting DNA. Science 322: $1843-1845$

Menon AL, Poole FL II, Cvetkovic A, Trauger SA, Kalisiak E, Scott JW, Shanmukh S, Praissman J, Jenney FE Jr, Wikoff WR, et al. 2009. Novel multiprotein complexes identified in the hyperthermophilic archaeon Pyrococcus furiosus by non-denaturing fractionation of the native proteome. Mol Cell Proteomics 8: 735-751.

Mojica FJ, Diez-Villaseñor C, Garcia-Martinez J, Soria E. 2005. Intervening sequences of regularly spaced prokaryotic repeats derive from foreign genetic elements. J Mol Evol 60: 174-182.

Mulepati S, Bailey S. 2013. In vitro reconstitution of an Escherichia coli RNA-guided immune system reveals unidirectional, ATP-dependent degradation of DNA target. J Biol Chem 288: 22184-22192.

Mulepati S, Heroux A, Bailey S. 2014. Structural biology. Crystal structure of a CRISPR RNA-guided surveillance complex bound to a ssDNA target. Science 345: 1479-1484.

Nam KH, Haitjema C, Liu X, Ding F, Wang H, Delisa MP, Ke A. 2012. Cas5d protein processes pre-crRNA and assembles into a cascadelike interference complex in subtype I-C/Dvulg CRISPR-Cas system. Structure 20: 1574-1584.
Pawluk A, Bondy-Denomy J, Cheung VH, Maxwell KL, Davidson AR. 2014. A new group of phage anti-CRISPR genes inhibits the type I-E CRISPR-Cas system of Pseudomonas aeruginosa. MBio 5: e00896.

Peng W, Li H, Hallstrom S, Peng N, Liang YX, She Q. 2013. Genetic determinants of PAM-dependent DNA targeting and pre-crRNA processing in Sulfolobus islandicus. RNA Biol 10: 738-748.

Peng W, Feng M, Feng X, Liang YX, She Q. 2015. An archaeal CRISPR type III-B system exhibiting distinctive RNA targeting features and mediating dual RNA and DNA interference. Nucleic Acids Res 43: 406-417.

Plagens A, Tjaden B, Hagemann A, Randau L, Hensel R. 2012. Characterization of the CRISPR/Cas subtype I-A system of the hyperthermophilic crenarchaeon Thermoproteus tenax. J Bacteriol 194: 2491-2500.

Plagens A, Tripp V, Daume M, Sharma K, Klingl A, Hrle A, Conti E, Urlaub H, Randau L. 2014. In vitro assembly and activity of an archaeal CRISPR-Cas type I-A Cascade interference complex. Nucleic Acids Res 42: 5125-5138.

Pourcel C, Salvignol G, Vergnaud G. 2005. CRISPR elements in Yersinia pestis acquire new repeats by preferential uptake of bacteriophage DNA, and provide additional tools for evolutionary studies. Microbiology 151: 653-663.

Ramia N, Spilman M, Tang L, Shao Y, Elmore J, Hale C, Cocozaki A, Bhattacharya N, Terns R, Terns M, et al. 2014. Essential structural and functional roles of the Cmr4 subunit in RNA cleavage by the Cmr CRISPR-Cas complex. Cell Rep 9: 1610-1617.

Reeks J, Naismith JH, White MF. 2013. CRISPR interference: a structural perspective. Biochem J 453: 155-166.

Santangelo TJ, Cubonova L, James CL, Reeve JN. 2007. TFB1 or TFB2 is sufficient for Thermococcus kodakaraensis viability and for basal transcription in vitro. J Mol Biol 367: 344-357.

Sashital DG, Wiedenheft B, Doudna JA. 2012. Mechanism of foreign DNA selection in a bacterial adaptive immune system. Mol Cell 46: 606-615.

Shevchenko A, Wilm M, Vorm O, Mann M. 1996. Mass spectrometric sequencing of proteins silver-stained polyacrylamide gels. Anal Chem 68: 850-858.

Shockley KR, Ward DE, Chhabra SR, Conners SB, Montero CI, Kelly RM. 2003. Heat shock response by the hyperthermophilic archaeon Pyrococcus furiosus. Appl Environ Microbiol 69: 2365-2371.

Sinkunas T, Gasiunas G, Waghmare SP, Dickman MJ, Barrangou R, Horvath P, Siksnys V. 2013. In vitro reconstitution of Cascade-mediated CRISPR immunity in Streptococcus thermophilus. EMBO J 32: 385-394.

Spilman M, Cocozaki A, Hale C, Shao Y, Ramia N, Terns R, Terns M, Li H, Stagg S. 2013. Structure of an RNA silencing complex of the CRISPR-Cas immune system. Mol Cell 52: 146-152.

Staals RH, Agari Y, Maki-Yonekura S, Zhu Y, Taylor DW, van Duijn E, Barendregt A, Vlot M, Koehorst JJ, Sakamoto K, et al. 2013. Structure and activity of the RNA-targeting Type III-B CRISPRCas complex of Thermus thermophilus. Mol Cell 52: 135-145.

Tang TH, Bachellerie JP, Rozhdestvensky T, Bortolin ML, Huber H, Drungowski M, Elge T, Brosius J, Huttenhofer A. 2002. Identification of 86 candidates for small non-messenger RNAs from the archaeon Archaeoglobus fulgidus. Proc Natl Acad Sci 99: 75367541.

Terns MP, Terns RM. 2011. CRISPR-based adaptive immune systems. Curr Opin Microbiol 14: 321-327.

Terns RM, Terns MP. 2013. The RNA- and DNA-targeting CRISPRCas immune systems of Pyrococcus furiosus. Biochem Soc Trans 41: $1416-1421$.

van der Oost J, Westra ER, Jackson RN, Wiedenheft B. 2014. Unravelling the structural and mechanistic basis of CRISPR-Cas systems. Nat Rev Microbiol 12: 479-492.

van Duijn E, Barbu IM, Barendregt A, Jore MM, Wiedenheft B, Lundgren M, Westra ER, Brouns SJ, Doudna JA, van der Oost J, et al. 2012. Native tandem and ion mobility mass spectrometry highlight structural and modular similarities in CRISPR-associated 
protein complexes from Escherichia coli and Pseudomonas aeruginosa. Mol Cell Proteomics 11: 1430-1441.

Vestergaard G, Garrett RA, Shah SA. 2014. CRISPR adaptive immune systems of Archaea. RNA Biol 11: 156-167.

Wang R, Preamplume G, Terns MP, Terns RM, Li H. 2011. Interaction of the Cas6 riboendonuclease with CRISPR RNAs: recognition and cleavage. Structure 19: 257-264.

Wells L, Vosseller K, Cole RN, Cronshaw JM, Matunis MJ, Hart GW. 2002. Mapping sites of $O$-GlcNAc modification using affinity tags for serine and threonine post-translational modifications. Mol Cell Proteomics 1: 791-804.

Westra ER, Nilges B, van Erp PB, van der Oost J, Dame RT, Brouns SJ. 2012a. Cascade-mediated binding and bending of negatively supercoiled DNA. RNA Biol 9: 1134-1138.

Westra ER, van Erp PB, Kunne T, Wong SP, Staals RH, Seegers CL, Bollen S, Jore MM, Semenova E, Severinov K, et al. 2012b. CRISPR immunity relies on the consecutive binding and degradation of negatively supercoiled invader DNA by Cascade and Cas3. Mol Cell 46: 595-605.

Wiedenheft B, Lander GC, Zhou K, Jore MM, Brouns SJ, van der Oost J, Doudna JA, Nogales E. 2011a. Structures of the RNA-guided sur- veillance complex from a bacterial immune system. Nature 477: 486-489.

Wiedenheft B, van Duijn E, Bultema JB, Waghmare SP, Zhou K, Barendregt A, Westphal W, Heck AJ, Boekema EJ, Dickman MJ, et al. 2011b. RNA-guided complex from a bacterial immune system enhances target recognition through seed sequence interactions. Proc Natl Acad Sci 108: 10092-10097.

Wiedenheft B, Sternberg SH, Doudna JA. 2012. RNA-guided genetic silencing systems in bacteria and archaea. Nature 482: 331-338.

Yosef I, Goren MG, Kiro R, Edgar R, Qimron U. 2011. High-temperature protein $\mathrm{G}$ is essential for activity of the Escherichia coli clustered regularly interspaced short palindromic repeats (CRISPR)/Cas system. Proc Natl Acad Sci 108: 20136-20141.

Zhang J, Rouillon C, Kerou M, Reeks J, Brugger K, Graham S, Reimann J, Cannone G, Liu H, Albers SV, et al. 2012. Structure and mechanism of the CMR complex for CRISPR-mediated antiviral immunity. Mol Cell 45: 303-313.

Zhao H, Sheng G, Wang J, Wang M, Bunkoczi G, Gong W, Wei Z, Wang Y. 2014. Crystal structure of the RNA-guided immune surveillance Cascade complex in Escherichia coli. Nature 15: $147-150$. 

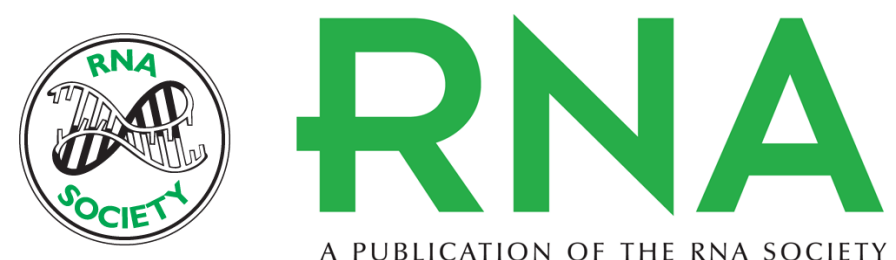

A PUBLICATION OF THE RNA SOCIETY

\title{
Three CRISPR-Cas immune effector complexes coexist in Pyrococcus furiosus
}

\author{
Sonali Majumdar, Peng Zhao, Neil T. Pfister, et al.
}

RNA 2015 21: 1147-1158 originally published online April 22, 2015

Access the most recent version at doi:10.1261/rna.049130.114

\section{Supplemental http://rnajournal.cshlp.org/content/suppl/2015/04/03/rna.049130.114.DC1 Material \\ References This article cites 76 articles, 30 of which can be accessed free at: http://rnajournal.cshlp.org/content/21/6/1147.full.html\#ref-list-1 \\ Creative This article is distributed exclusively by the RNA Society for the first 12 months after the Commons License full-issue publication date (see http://rnajournal.cshlp.org/site/misc/terms.xhtml). After 12 months, it is available under a Creative Commons License (Attribution-NonCommercial 4.0 International), as described at http://creativecommons.org/licenses/by-nc/4.0/.}
Email Alerting Receive free email alerts when new articles cite this article - sign up in the box at the Service top right corner of the article or click here.

To subscribe to RNA go to:

http://rnajournal.cshlp.org/subscriptions 